\title{
Fe I line shifts in the optical spectrum of the Sun ${ }^{\star}$
}

\author{
C. Allende Prieto and R.J. García López \\ Instituto de Astrofísica de Canarias, E-38200 La Laguna, Tenerife, Spain
}

Received August 5; accepted September 17, 1997

\begin{abstract}
New improvements in the measurement of both the optical solar spectrum and laboratory wavelengths for lines of neutral iron are combined to extract central wavelength shifts for 1446 lines observed in the Sun. This provides the largest available database of accurate solar wavelengths useful as a reference for comparison with other solar-type stars. It is shown how the velocity shifts correlate with line strength, approaching a constant value, close to zero, for lines with equivalent widths larger than $200 \mathrm{~m} \AA$.
\end{abstract}

Key words: convection - atlases - Sun: photosphere - stars: atmospheres

\section{Introduction}

Convective motions in the photosphere are nowadays believed to be the main contributors to observed line asymmetries and shifts in the optical spectra of the Sun and solar-type stars. Several studies have been devoted to the analysis and classification of these observational features in the Sun (e.g. Dravins et al. 1981, hereafter DLN; Balthasar 1984). Some researchers, extending this work to other stars, have seen how the difficulties grow, not only due to the lack of photons but also to the impossibility of accurately removing two major effects: gravitational shifts and the radial velocity of the star, which in turn do not allow the setting up of an absolute velocity scale (e.g. Gray 1982; Dravins 1987a,b). However, absolute line shifts should be included in spectral syntheses and inversion codes that take velocity patterns into account. Line asymmetries and shifts establish a footprint of the dynamics of convection, thereby imposing a major constraint to the theoretical modelling.

The increasing resolving power of the spectrographs and the improvements of detectors have made systematic high resolution spectroscopic observations possible over

Send offprint requests to: C. Allende Prieto (callende@iac.es)

* Table 1 is available in electronic form only via the CDS ftp 130.79.128.5 or http://cdsweb.u-strasbg.fr/Abstract.html wide spectral ranges. Recent studies (e.g. Allende Prieto et al. 1995) have extended very high-quality spectroscopic observations beyond the domain of the brightest stars. In the solar case, optical, IR and UV atlases are available from modern observations with very high signal-to-noise ratio and spectral resolution. For the optical spectrum of the Sun seen as a star, the Solar Flux Atlas from 296 to $1300 \mathrm{~nm}$ (Kurucz et al. 1984, referred to here as the FTS flux spectrum) has been the most extensively used. Partially from the same data set (flux), partially from a second set of Kitt Peak FTS spectra (disc-centre intensity), H. Neckel prepared the Spectral Atlas of Solar Absolute Disk-Averaged and Disk-Center Intensity from 3290 to $12510 \AA$ (Brault \& Neckel 1987; for details see Neckel 1994). We shall refer to the included disc-centre spectrum as the FTS disc-centre spectrum. These atlases achieve signal-to-noise ratios of about 2500 and a resolving power $\lambda / \Delta \lambda \sim 400000$.

The set of lines with wavelengths accurate enough to be useful in this context has been significantly enlarged by the work of Nave et al. (1994), who measured and identified $9501 \mathrm{Fe}$ I lines using Fourier transform spectrometers at $\mathrm{NSO}^{1}$, Imperial College (London, UK), and $\mathrm{NIST}^{2}$, from 0.17 to $5 \mu \mathrm{m}$. In the optical range, they claim an uncertainty below $1 \mathrm{~m} \AA$ for many lines, and two orders of magnitude larger for the worst cases.

We have used these relatively new atlases, as well as the more traditional Liège Atlas (Delbouille et al. 1973), to measure central wavelengths for a large subsample of the neutral iron lines included in the line list by Thèvenin (1989, 1990). His compilation joins those lines classified by Moore et al. (1966) as one-blended or unblended, and it was taken as a starting point since the determination of accurate line centres does not require such a clean profile as is needed to measure line asymmetries. This information is combined with the rest air wavelengths from Nave et al. (1994) to deduce the displacements of the solar lines.

\footnotetext{
1 National Solar Observatory, Tucson, Arizona, U.S.A. 2 National Institute of Standards and Technology, Gaithersburg, Maryland, U.S.A.
} 


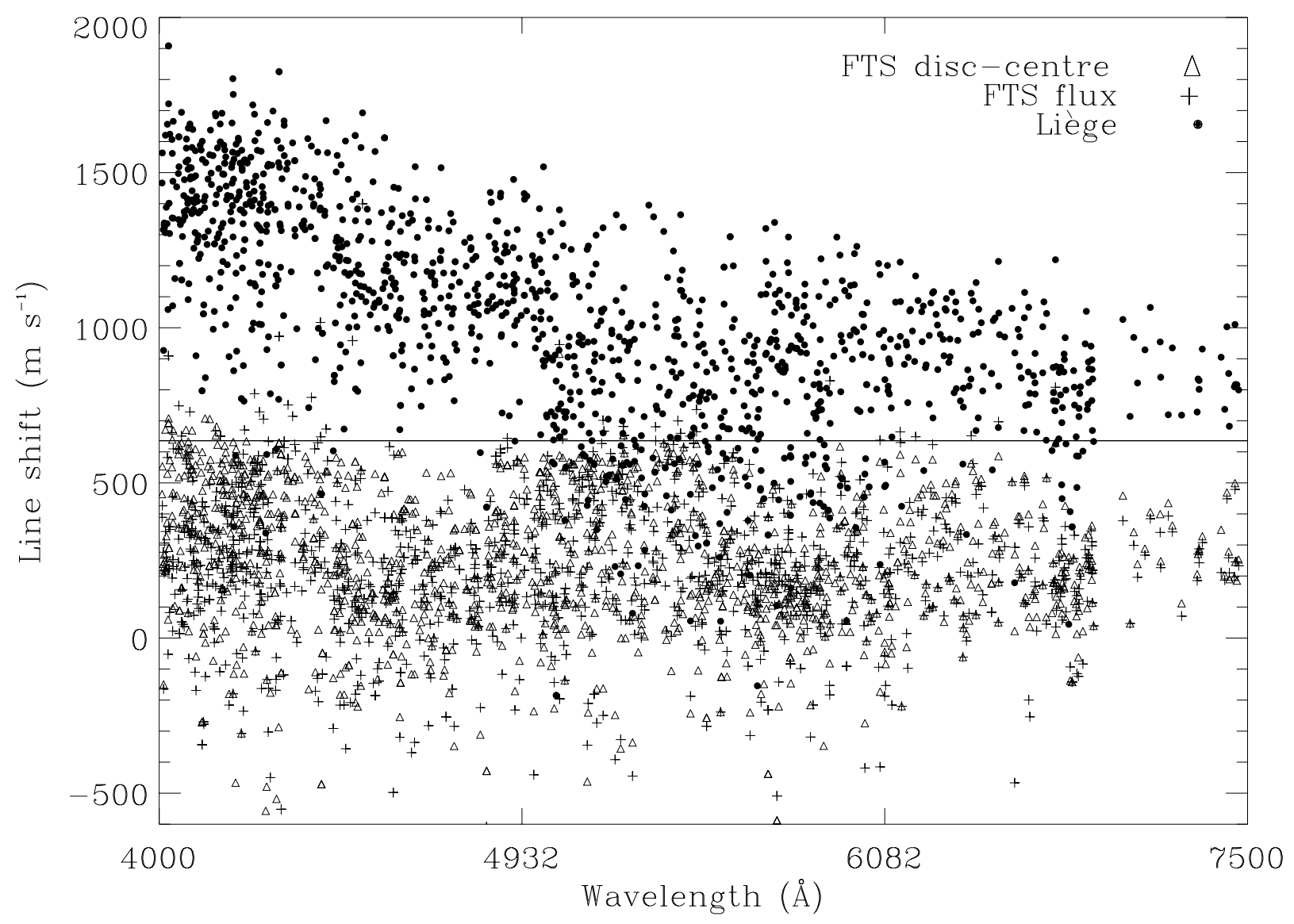

Fig. 1. Line shifts measured in the FTS disc-centre spectrum (triangles) and in the FTS flux spectrum (plus signs) show no trend with wavelength. The largest shifts to the red are consistent with the expected gravitational shift of $636 \mathrm{~m} \mathrm{~s}^{-1}$ (solid line). The shifts measured in the Liège Atlas (dots) exhibit a wavelength dependence, and go further to the red than the gravitational redshift, thereby revealing calibration errors

\section{Absolute wavelength calibration of the atlases}

As a first step, the wavelength calibrations of the two FTS atlases mentioned above were tested. This was also done for the Liège Atlas, which is composed of disc-centre observations performed at Jungfraujoch Observatory (Swiss Alps) using a double-pass grating spectrometer, and which has been extensively used in many solar studies. We have made use of the version available as part of the $\mathrm{KIS}^{3}$ IDL library.

It has been shown (e.g. Balthasar 1984) that once the Sun-Earth velocity shifts have been corrected for, the maximum displacement to the red exhibited by solar spectral lines corresponds to the gravitational redshift due to the difference in gravity between the solar and terrestrial surfaces. This is the expected case for the three atlases under consideration, which use wavelengths on standard air (dry, $\left.15{ }^{\circ} \mathrm{C}, 760 \mathrm{mmHg}\right)$. Solar-Earth doppler shifts were corrected in the FTS flux spectrum, and the same is true for the tables of solar wavelengths prepared by Pierce \&

\footnotetext{
3 Kiepenheuer-Institut für Sonnenphysik, Freiburg, Germany.
}

Breckenridge (1973) at Kitt Peak, which were employed to calibrate the wavelength scale of the FTS disc-centre spectrum. Both FTS spectra were compiled from eight (flux) or seven (disc-centre) carefully overlapped scans. The Liège atlas is supposed to be calibrated following the same reference, but prior to publication.

Following the procedure described in the next section, shifts for neutral iron lines were measured in the atlases. Figure 1 shows the results as a function of wavelength. It appears that both the FTS disc-centre spectrum and the FTS flux spectrum agree in the absolute scale within the errors and the expected differences between intensity and flux measurements. They do not show any stronger than expected trend (Hamilton 1997) and are in agreement with the expected maximum redshift of $636 \mathrm{~m} \mathrm{~s}^{-1}$ (solid line), which corresponds to the gravitational effect. On the contrary, the wavelength calibration of the Liège Atlas differs clearly on the absolute scale, exhibiting redshifts larger than $636 \mathrm{~m} \mathrm{~s}^{-1}$ and showing a strong trend with wavelength, which points towards errors in the spectral calibration procedure. For this reason we have discarded the Liège Atlas in this study. 


\section{Line shifts}

\subsection{Intensity}

From the most extensive up-to-date measurements of solar wavelengths by Pierce \& Breckenridge (1973), DLN extracted a list of 311 unblended Fe I lines. After subtracting the gravitational redshift, they studied the trend of the shifts with the excitation potential of the lines and placed the bisectors measured in the solar atlas of Liège Atlas on an absolute scale. Their study demonstrated the power of line asymmetries and shifts in providing a deeper understanding of solar convection.

A version of the FTS disc-centre spectrum, which has been interpolated to a constant wavelength step, can be obtained as part of the KIS IDL library. We have measured on this atlas the location of the minima of 1446 Fe I lines selected from the list of Thévenin (1989, 1990), thereby extending the existing data provided by DLN and improving its accuracy significantly. Thèvenin's list includes 2536 lines of neutral iron. Although many of the lines are blended, only those showing clear evidence for the blend to disturb the line centre were rejected. A fourth-order polynomial was fitted to the $50 \mathrm{~m} \AA$ wavelength interval around the line bottom to find the line centre as precisely as possible. Measured central wavelengths, together with their corresponding air values at rest, excitation potentials, transition probabilities and equivalent widths, are listed in Table 1. Since the measurement process was automatic, Table 1 may contain certain errors for the observed wavelengths.

The centres of those lines formed higher in the photosphere show smaller blueshifts. It can be seen in Fig. 2a that even those lines located in a "plateau" with equivalent widths $\left(W_{\lambda}\right)$ larger than $\sim 100 \mathrm{~m} \AA$ seem to be somewhat blueshifted, and there are no lines where the effect is negligible. The mean ${ }^{4}$ value for the shift of these lines is $539 \mathrm{~m} \mathrm{~s}^{-1}$ (subtracting the gravitational redshift of $636 \mathrm{~m} \mathrm{~s}^{-1}$, the minimum convective blueshift will be $97 \mathrm{~m} \mathrm{~s}^{-1}$ ) with a standard deviation of $86 \mathrm{~m} \mathrm{~s}^{-1}$. No other source in the literature, including spectra and wavelength measurements, is accurate enough to perform a more reliable comparison discarding minor systematic effects of the wavelength calibration.

\subsection{Flux}

To the best of our knowledge, the only existing measurements of line shifts from solar flux spectra are those of Burns, Meggers, and Kiess, published in 1929 (Burns 1929; Burns \& Kiess 1929; Burns \& Meggers 1929). Nonetheless, systematic analyses of shifts and asymmetries of spectral lines in the Sun seen as a star are of special importance as a reference standard for comparison with other stars.

\footnotetext{
4 Only lines without an asterisk in the equivalent widths shown in Table 1 were included.
}

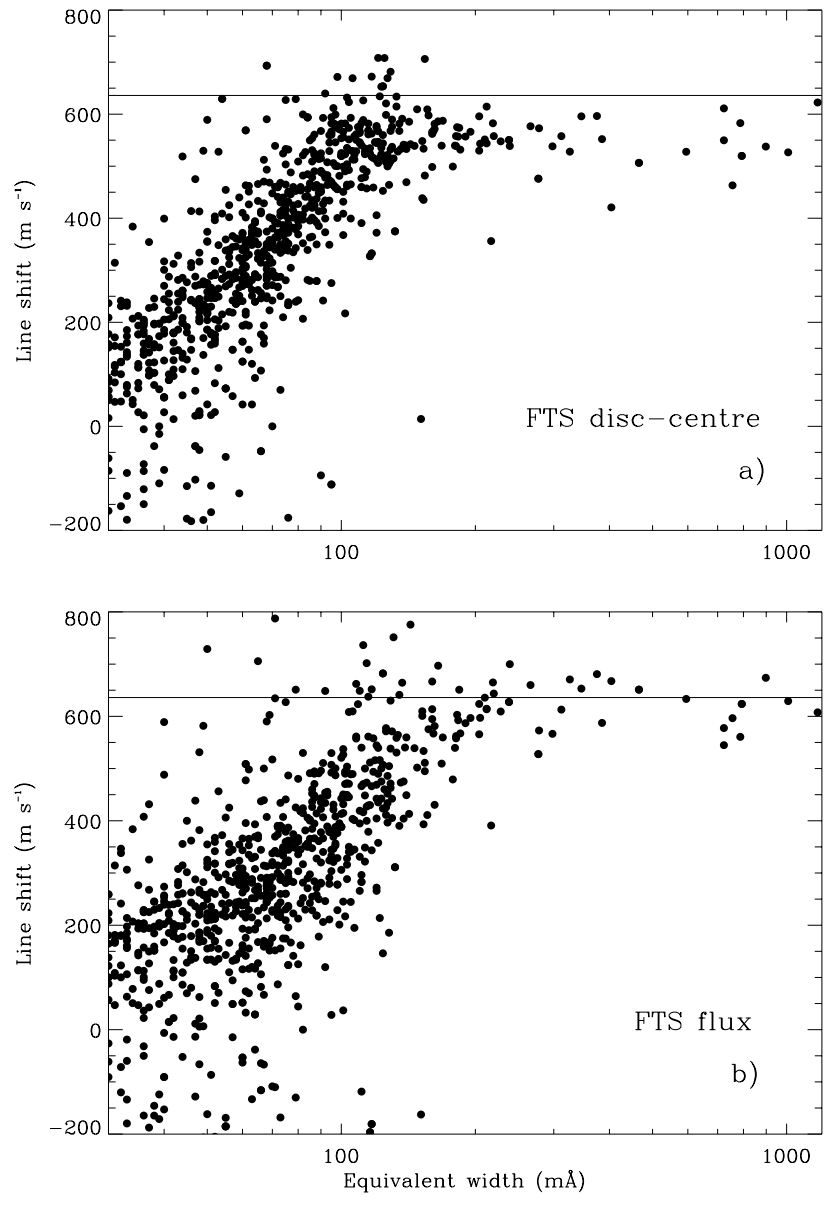

Fig. 2. a and b). Line shifts as measured in the FTS disc-centre spectrum a) and the FTS flux spectrum b) are plotted as a function of the equivalent width at the centre of the disc (Moore et al. 1966). Known blends (marked with an asterisk in Table 1) have been rejected

The FTS flux spectrum is available from the $\mathrm{NOAO}^{5}$ $\mathrm{ftp}$ site. We have measured the wavelengths of the minima of the same 1446 Fe I lines considered in the FTS disccentre spectrum. Similarly to the intensity case, a fourthorder polynomial was fitted to the $55 \mathrm{~m} \AA$ wavelength interval around the line centre. The results are included in the second column of Table 1 . This list provides the largest available set of accurate solar wavelengths useful as a reference for comparison with other solar-type stars.

The smoothing of the convective blueshift towards the solar limb, the so-called limb effect, results in a smoothing of the convective shifts in the integrated sunlight compared with the intensity spectrum. In this atlas, the "plateau" with equivalent widths larger that $\sim 200 \mathrm{m \AA}$ is formed by lines distributed around the gravitational redshift, as can be seen in Fig. $2 \mathrm{~b}$. It makes sense that the smoothing of the blue-shifts at the disc centre results in a plateau closer to a null velocity shift. The scatter

5 National Optical Astronomical Observatories, U.S.A. 
(standard deviation) in these line shifts at the plateau is $58 \mathrm{~m} \mathrm{~s}^{-1}$, around a mean value of $612 \mathrm{~m} \mathrm{~s}^{-1}$ (the subtraction of the gravitational redshift comes with a mean of $24 \mathrm{~m} \mathrm{~s}^{-1}$ for the smaller convective blueshifts).

Wallace, Huang \& Livingston (1988) studied the variability of the convective line shifts in the solar flux spectrum during the solar cycle measuring the relative shifts between a weak line, $\mathrm{C}_{\text {I }} \lambda 5380.3 \AA\left(W_{\lambda}=26 \mathrm{~m} \AA\right)$ and the stronger features Fe I $\lambda 5379.6 \AA\left(W_{\lambda}=67 \mathrm{~m} \AA\right)$ and Ti II $\lambda 5381.0 \AA\left(W_{\lambda}=70 \mathrm{~m} \AA\right)$. They found an upper limit of $5 \mathrm{~m} \mathrm{~s}^{-1}$ for the relative line shift and concluded with the possibility of detecting Jupiter, which would produce a $20 \mathrm{~m} \mathrm{~s}^{-1}$ amplitude in the solar radial velocity, from an extra-solar system observer. However, Fig. 2b suggests that the $\sim 70 \mathrm{~m} \AA$ pair of lines they employed as reference wavelengths less affected by convection were not the ideal choice. Comparison between a much stronger feature and the $\mathrm{C}$ I line will probably give a safer answer on this subject, where different measurement techniques observe (Deming \& Plymate 1994) and deny (McMillan et al. 1993) the variations of the convective shifts.

\section{Conclusions}

We have verified that the line wavelength shifts measured in the FTS disc-centre spectrum and the FTS flux spectrum agree on an absolute scale and do not show any strong trend with wavelength, whereas the Liège Atlas exhibits an anomalous wavelength dependence with line shifts in excess of the gravitational redshift, thereby revealing calibration errors.

Accurate wavelengths have been measured for $1446 \mathrm{Fe}$ I lines, both from intensity and flux spectra, providing the largest database available for comparison with other solartype stars. This line list may be useful in employing solar (daylight or lunar) spectra to perform wavelength calibrations in high-resolution spectroscopy, or to assess the quality of calibrations based on spectral lamps, which normally illuminate the detector through a different optical path from that of the observational target.

Finally, it has been shown how the wavelength shifts of lines formed at the top of the photosphere get close to the gravitational redshift.

Acknowledgements. We thank H. Neckel for the help in dealing with his solar atlas, F. Thèvenin and A.D. Wittmann for providing us with a copy of their digital solar line lists, and H.H.R. Kroll for installing and maintaining the KIS computer libraries at the IAC. We are grateful to J. Sánchez Almeida for interesting comments after careful reading of the manuscript. Valuable bibliographic information was pointed out to us by the referee. NSO/Kitt Peak FTS data used here were produced by NSF/NOAO.

This work was partially supported by the Spanish DGES under projects PB92-0434-C02-01 and PB95-1132-C02-01.

\section{References}

Allende Prieto C., García López R.J., Lambert D.L., Gustafsson B., 1995, in Stellar Surface Structure, IAU Symp. 176: Poster Proceedings, Strassmeier K.G. (ed.), Vienna: Institut für Astronomie der Universität Wien, p. 107

Balthasar H., 1984, Solar Phys. 93, 219B

Brault J., Neckel H., 1987, Spectral Atlas of Solar Absolute Disk-Averaged and Disk-Center Intensity from 3290 to $12510 \AA$, unpublished (tape-copy from KIS IDL library)

Burns K., 1929, Publ. Allegheny Obs. 6, 141

Burns K., Kiess C.C., 1929, Publ. Allegheny Obs. 6, 125

Burns K., Meggers W.F., 1929, Publ. Allegheny Obs. 6, 105

Delbouille L., Neven L., Roland G., 1973, Photometric Atlas of the Solar Spectrum from $\lambda 3000$ to $\lambda 10000$, Liège

Deming D., Plymate C., 1994, ApJ 426, 382

Dravins D., 1987a, A\&A 172, 200

Dravins D., 1987b, A\&A 172, 211

Dravins D., Lindegren L., Norlund Å, 1981, A\&A 96, 345 (DLN)

Gray D.F., 1982, ApJ 255, 200

Hamilton D., 1997, poster paper to appear in the proceedings of the $10^{\text {th }}$ Cambridge Workshop on Cool Stars, Stellar Systems and the Sun

Kurucz R.L., Furenlid I., Brault J., Testerman L., 1984, NOAO Atlas No. 1, The Solar Flux Atlas from 296 to $1300 \mathrm{~nm}$, Sunspot, NM: National Solar Observatory

McMillan R.S., Moore T.L., Perry M.L., Smith P.H., 1993, ApJ 403, 801

Moore E., Minnaert M.G.J., Houtgast J., 1966, The Solar Spectrum $2935 \AA$ to $8770 \AA$, National Bureau of Standards Monograph 61

Nave G., Johansson S., Learner R.C.M., Thorne A.P., Brault J.W., 1994, ApJS 94, 221

Neckel H., 1994, in The Sun as a Variable Star, IAU Coll. 143, p. 37

Pierce A.K., Breckinridge J.B., 1973, Kitt Peak National Observatory, Contribution No. 559

Thèvenin F., 1989, A\&AS 77, 137

Thèvenin F., 1990, A\&AS 82, 179

Wallace L., Huang Y.R., Livingston W., 1988, ApJ 327, 399 